\title{
VII Programa General de Acción de la Unión Europea en materia de Medio Ambiente 2014-2020 y Huerta de Valencia
}

\author{
Antonio Montiel Márquez y Eduardo García de Leonardo Tobarra \\ Universitat de València - Estudi General \\ Antonio.Montiel@uv.es,Eduardo.Garcia-Tobarra@uv.es
}

\begin{abstract}
Resumen. En esta ponencia se trata de relacionar alguno de los contenidos del VII Programa General de Medio Ambiente 2014-2020, aprobado en el contexto de la Estrategia Europa 2020, así como de la nueva regulación de los instrumentos de la Unión Europea, con el fin de explorar su posible incidencia en las políticas en favor de acciones y medidas para la preservación de los suelos de l'Horta de Valencia como espacio agrícola periurbano y como matriz necesaria y fundamental para una correcta articulación de la infraestructura verde del área metropolitana de Valencia.
\end{abstract}

Palabras clave: Huerta de Valencia, agricultura periurbana, VII Programa General de Medio Ambiente, Fondos Estructurales 2014-2020.

\begin{abstract}
With this report we intend to link some of the contents of the VII General Environment Programme 2014-2020, as well as the new European Union financial instruments regulation in order to explore its posible impact on the policies in favor of actions and measures for the soil preservation of the Horta de Valencia as a peri-urban agricultural espace and as a necessary and essential matrix for a correct articulation of the green infraestructura of the metropolitana area.
\end{abstract}

Keywords: Huerta de Valencia, peri-urban agriculture, VII General Environment Programme, Structural Founds 2014-2020.

\section{Introducción. El valor del recurso suelo en la Huerta de Valencia}

En 1995 la Agencia Europea de Medio Ambiente (AEMA) publicó el informe "El Medio Ambiente en Europa" (Informe Dobris), que constituyó en su momento el estudio más minucioso y exhaustivo sobre la situación del medio ambiente en Europa, con datos obtenidos hasta 1992 procedentes de numerosas fuentes y abarcando a 46 países. La revisión posterior de este informe (Dobris + 3) clasificaba 30 paisajes en Europa. Uno de estos paisajes se denominaba específicamente con el término español Huerta y corresponde a tan sólo seis pequeños ámbitos situados en Italia, Grecia y España. Son los fragmentos más reducidos en extensión del total paisajístico y son reflejo de una cultura mediterránea milenaria.

El informe situaba dos de esos seis enclaves agrarios históricos en España, ambos seriamente amenazados ${ }^{1}$, las Huertas de Valencia y Murcia.

Este reconocimiento del valor de l'Horta y de las amenazas que se cernían sobre ella estuvo también en la base del Dictamen adoptado en mayo de 2000 por el Consell Valencià de Cultura y de la misma Iniciativa Legislativa Popular promovida por colectivos ciudadanos

1 La propia Estrategia Territorial de la Comunitat Valenciana, aprobada por Decreto 1/2011, de 13 de enero, del Consell de la Generalitat Valenciana (DOCV 6645, de 07.11.2011), a la que luego nos referiremos más extensamente, señala la desaparición de $1.500 \mathrm{Ha}$ de huerta en los últimos 20 años, si bien trabajos realizados por entidades independientes como el colectivo ciudadano Per l'Horta, apuntan a cifras muy superiores (www.perlhorta.info). 
en el año 2001 que, aun habiendo sumado más del doble de las firmas exigidas para su tramitación parlamentaria, fue rechazada por el parlamento valenciano ${ }^{2}$.

Sin embargo, el debate social y la movilización ciudadana, dieron como fruto, aunque tardío, la adopción por la Generalitat Valenciana de diversas medidas tendentes a la protección siquiera sobre el papel de l'Horta. Una de estas medidas fue la inclusión de un mandato legal en el artículo 22.6 de la Ley 4/2004, de 30 de junio, de la Generalitat, de Ordenación del Territorio y Protección del Paisaje (LOTPP), a fin de que el Consell aprobase un Plan de Acción Territorial de Protección de la Huerta Valenciana. Otra medida fue la declaración como Bien de Interés Cultural Inmaterial del Tribunal de las Aguas de la Huerta de Valencia, mediante el Decreto 73/2006, de 26 de mayo, del Consell y, meses más tarde y mediante el Decreto 148/2006, de 6 de octubre, la declaración como Bienes de Interés Cultural, con la categoría de monumento, los Azudes de las Acequias del Tribunal de las Aguas de Valencia y de la Real Acequia de Moncada, situados en Valencia, Paterna, Quart de Poblet y Manises, así como la declaración del Conjunto Histórico que forman los mismos.

El primero de dichos Decretos, en el último párrafo de su anexo, constataba la siguiente evidencia:

La conservación del Tribunal de las Aguas de la Huerta de Valencia está supeditada al mantenimiento de las comunidades de regantes, y a la práctica de la agricultura tradicional de regadio en la Huerta de Valencia, por lo cual, la Generalitat, en coordinación con las entidades locales implicadas y las comunidades de regantes, arbitrará las medidas oportunas para garantizar la pervivencia de esta ancestral institución. Estas se articularán a través del Plan de Acción Territorial de Protección de la Huerta de Valencia que prevé el artículo 22 de la Ley 4/2004, de 30 de junio, de la Generalitat, de Ordenación del Territorio y Protección del Paisaje"

El mandato referido en dichas normas se concretó en la redacción del denominado Plan de Acción Territorial de Protección de la Huerta de Valencia (PATPHV, en adelante), en cuya memoria informativa se podía leer:

El paisaje de la Huerta de Valencia es un patrimonio histórico, cultural, natural y agrícola del pueblo valenciano. Es el resultado de una integración armoniosa del hombre con su entorno durante generaciones, y constituye un paisaje irremplazable con una personalidad única. Hoy en día, la buerta de Valencia está desapareciendo y se enfrenta a condiciones y cambios socioeconómicos que ponen en riesgo su supervivencia.

Asimismo, según el Informe Dobris de 1998 de la European Environmental Agency (ver bibliografia) la Huerta de Valencia es una de las uiltimas 6 huertas mediterráneas históricas en Europa, todas ellas amenazadas de desaparición en la actualidad. Por tanto, la Huerta de $V$ alencia no sólo tiene interés local o regional, sino también universal.

Desgraciadamente el impulso político se extinguió pronto. En septiembre de 2010, tras recibir numerosas alegaciones de colectivos sociales, ciudadanos individuales y entidades de todo tipo, el PATPHV quedo arrumbado en algún despacho de la Generalitat sin que nunca más se supiese de él salvo por referencias académicas ${ }^{3}$.

\footnotetext{
2 Montiel Márquez (2012) ha tenido ocasión de analizar las funciones que desempeña l'Horta de València y las oportunidades actuales de la misma en varios trabajos. Vid. asimismo, Giobellina (2012) y Tortosa (2007).

3 Un análisis crítico del contenido del PATHV y de la posterior Estrategia Territorial de la Comunitat Valenciana, puede verse también en Montiel Márquez (2012).
} 


\section{El papel de la agricultura periurbana en las grandes aglomeraciones europeas.}

En septiembre de 2004, el Comité Económico y Social Europeo (CESE, en adelante), aprobó, sin ningún voto en contra, un Dictamen de iniciativa dedicado a la agricultura periurbana ${ }^{4}$ que constituye una referencia básica para el abordaje de esta cuestión. Los argumentos que le servían de justificación son plenamente actuales:

El CESE, como una de las partes interesadas en el desarrollo rural directamente empeñadas en asegurar la sostenibilidad del desarrollo económico, medioambiental y social de las zonas rurales de Europa, recuerda que dichas funciones medioambientales, sociales y económicas que también realizan los espacios agrarios tienen en las áreas periurbanas una mayor relevancia que en el resto del territorio. En estas áreas el suelo agrario actúa como pulmón verde de las grandes urbes; estas áreas son un elemento fundamental en la ordenación territorial que impiden el crecimiento ilimitado de las ciudades, generan paisaje y humanizan los entornos urbanos. Por el contrario, su función económica, fundamental para el mantenimiento y las perspectivas de futuro de los espacios agrarios, se ve reducida por la presión urbanistica que debe soportar y por la escasa importancia productiva que se le atribuye en el marco general de la economía de las áreas periurbanas.

El Dictamen, tras desarrollar la importancia de las diversas funciones que desempeñaba la agricultura periurbana, definía una serie de objetivos para su preservación y desarrollo, basado en la articulación de pactos ciudad-campo a través de órganos gestores, básicamente institucionales, y de mecanismos de participación ciudadana, y del sector agrario, con especial atención a la incorporación activa de mujeres y jóvenes en los proyectos territoriales y contratos agrarios.

Tales pactos habían de servir, según el CESE, a la consecución de tres objetivos:

a) Primer objetivo: la existencia de un proyecto territorial de preservación y desarrollo de los espacios con agricultura periurbana. Proyectos que deben articularse mediante planes territoriales, urbanisticos y uso del suelo, asi como legislaciones especificas que regulen el mercado del suelo agrario.

b) Segundo objetivo: la estabilidad del suelo agrario periurbano mediante instrumentos $y$ mecanismos que garanticen dicha estabilidad, reduciendo al máximo la presión urbanistica y los usos ajenos a la actividad agraria y facilitando el acceso al uso agrario de la tierra.

c) Tercer objetivo: una gestion integral mediante un ente gestor que actúe de impulsor y dinamizador del espacio agrario periurbano además de dar a conocer a la ciudadanía los valores de estos territorios periurbanos. Ello debe suponer la garantía de desarrollo dinámico y sostenible mediante un compromiso de gestión a partir de proyectos rururbanos y una relación contractual entre los ciudadanos, la administración y los agricultores, a partir de un contrato para la gestión agraria sostenible.

Señalándose como condiciones fundamentales para la consecución de tales objetivos, además de la ya referida implicación de mujeres y jóvenes en la actividad agraria y el cambio de percepción y reconocimiento social a las funciones de la actividad agrícola saludable y de proximidad, la mejora de los servicios para la actividad agrícola y el favorecimiento de su producción y comercialización con la finalidad de mejorar las rentas agrarias, lo que el Dictamen ya denomina una "nueva cultura del agua", como factor clave para la consolidación

${ }^{4}$ DOUE C74/62 de 23.03.2005 
de los espacios agrarios periurbanos. Así apuntaba que eran necesarias legislaciones específicas que, sin menoscabar el uso del agua para la agricultura, incorporasen esa nueva cultura, basada en el ahorro del uso de las aguas superficiales y subterráneas y la reutilización de las aguas procedentes de procesos de depuración de aguas residuales para usos agrarios.

Lo cierto es que las recomendaciones del CESE, a pesar de haber contribuido a alentar numerosas reflexiones e iniciativas posteriores, no ha obtenido la respuesta que cabía esperar en los Reglamentos comunitarios que conforman las líneas básicas de la nueva Política Agraria Común (PAC, en adelante) para el período 2014-20205, sin que de los aspectos clave para la aplicación de la reforma que quedan pendientes de decisión por parte del Estado Español como estado miembro de la Unión, quepa esperar alguna novedad significativa en orden a la protección y desarrollo de la agricultura periurbana, por contraste con la agricultura de montaña que no sólo ha seguido manteniendo su estatus como ámbito con necesidades específicas, objeto de un subprograma temático propio en la nueva PAC, sino que las ayudas asignadas en ésta pueden ser mejoradas por decisión de cada estado miembro.

Y ello, aún a pesar de que entre las preocupaciones expresadas en el debate europeo acerca de la cohesión territorial se haya insistido en la idea de una nueva asociación entre las zonas rurales y urbanas (Resolución del Parlamento Europeo de 24 de marzo de 2009, sobre la cohesión territorial y el estado del debate sobre la futura reforma de la política de cohesión $)^{6}$. Una idea para la que la agricultura periurbana en cuanto actividad radicada en la zona de transición, donde interaccionan dos formas de organizar, de entender y de vivir el territorio, podía constituir un magnífico ámbito de ensayo para esta nueva asociación. Una nueva evidencia de que las preocupaciones territoriales y las políticas agrícolas, tanto europeas como nacionales, suelen discurrir con obstinada frecuencia por caminos separados.

La realidad de la agricultura periurbana, sus funciones y especificidades, ha quedado así ausente de la "gran política", pero no así de las preocupaciones e inquietudes de una buena parte de la ciudadanía, prueba de lo cual lo constituyen los dos Congresos Estatales de agricultura ecológica urbana y periurbana, celebrados en mayo de 2011, "Huertos Urbanos y Desarrollo Sostenible", y marzo de 2014, "Huertos urbanos, autoconsumo y participación social", celebrados en Elx (Alicante) y Utrera (Sevilla), respectivamente.

Con todo, la iniciativa más significativa la constituye la denominada Carta de la Agricultura Periurbana adoptada en el Seminario Agroterritorial, celebrado en Castelldefels (Barcelona) los días 8 y 9 de septiembre de 2010, a convocatoria del Consorci del Parc Agrari del Baix Llobregat, por la Fundació Agroterritori y la Red Agroterritorial en el marco del proyecto AGRI-PROXI «La agricultura de proximidad para re-inventar las relaciones entre los espacios urbanos y rurales», correspondiente al Programa Operativo de Cooperación Territorial España-Francia-Andorra 2007-2013 (POCTEFA). Carta suscrita además a título personal por una cincuentena de destacados expertos y académicos.

En esta declaración se definen con interesante detalle una serie de concretas actuaciones en diversos ámbitos (el reconocimiento social, económico, profesional y científico, de la protección y gestión territorial, de la gobernanza, de la gestión agronómica y de la

${ }^{5}$ DOUE, L 347, de 20.12.2013.

${ }^{6}$ Sobre el concepto de cohesión territorial, vid. Camacho Ballesta y Melikhova (2010) y Farinós Dasí (2009). 
comercialización, del paisaje y del patrimonio cultural y de la biodiversidad) para la consecución de los siguientes objetivos:

“a) Que la especificidad de las zonas agricolas periurbanas y de la relación ciudad-campo tenga un reconocimiento explícito, en los ámbitos social, político y administrativo, a partir de normas, acciones y medidas propias destinadas a esta agricultura.

b) Que los municipios con presencia de agricultura periurbana, en especial en los ámbitos metropolitanos, dispongan de instrumentos eficaces para la planificación, la preservación y la gestión de sus espacios agrarios periurbanos, a la vez que fomenten y apliquen las legislaciones y medidas necesarias para ordenar el territorio y posibilitar el acceso a la tierra de aquellos que se incorporen a la actividad agraria y de aquellos que necesiten incrementar la base territorial de sus explotaciones agrarias.

c) Que la agricultura periurbana sea entendida, estudiada, analizada, planificada y gestionada desde una visión supramunicipal de conjunto, evitando las prácticas localistas excluyentes, con la finalidad de establecer una política pública más eficazy más eficiente dirigida a los espacios agrarios periurbanos.

d) Que los suelos periurbanos de valor agrícola se clasifiquen como suelo agricola, con independencia de que estén o no en explotación, entendiendo que "suelo agrícola" es una categoría finalista, del mismo modo que lo es la de "suelo urbano".

e) Que se garantice, más allá de la preservación del espacio agrario a través de los instrumentos urbanisticos, el desarrollo dinámico y sostenible de la actividad agraria periurbana y de los territorios donde esta se desarrolla con políticas especificas territoriales y sectoriales.

f) Que se incorpore la figura de "parque agrario" u otras figuras de ordenación y gestion adaptadas a cada realidad como elementos significativos de la voluntad para dotar a estos espacios de un proyecto, no sólo de protección frente a su eventual incorporación al proceso urbanizador, sino de preservación de las funciones agrarias propias y de impulso a una gestión que permita promover en ellos el desarrollo económico del territorio y de las explotaciones agrarias, así como conservar y difundir los valores ecológicos y culturales".

Una declaración que, por lo que parece, no ha estimulado hasta la fecha iniciativa institucional alguna.

\section{La incipiente protección del recurso suelo en el marco de la política comunitaria de medio ambiente}

La protección del recurso natural del suelo, en cuanto recurso vital y no renovable, la encontramos inicialmente en la Carta Europea del Suelo del Consejo de Europa (1972). También, en el plano internacional, en la Carta Mundial de los Suelos de la FAO de 1982 y, más recientemente, en los acuerdos de Río 2012 sobre la buena gestión de las tierras y el suelo y el compromiso para evitar su degradación.

En el marco de la política comunitaria de medio ambiente, se viene abriendo tímidamente en estos últimos años una línea de trabajos y actuaciones dirigidos a la protección del recurso suelo.

Un elemento fundamental de la política comunitaria de medio ambiente han sido los sucesivos Programas de Acción en Materia de Medio Ambiente (artículo 192 TFUE), que han proporcionado el marco para la actuación medioambiental de la Unión desde 1973. 
El Sexto Programa (2002-2010) ${ }^{7}$ destacó el papel de la planificación y la gestión de los usos del suelo a escala local y regional y se refirió, entre otras muchas cuestiones, al papel de la planificación y gestión de los usos del suelo, si bien reconocía que "el unico papel que puede desempeñar la Comunidad es impulsar y propiciar una planificación eficazy políticas adecuadas a nivel local y regional".

Dicho Programa preveía también la elaboración y puesta en práctica de una serie de estrategias temáticas que respondiesen a una serie de temas complejos que exigen un enfoque amplio y multidimensional. De este modo, se elaboraron y adoptaron por la Comisión una serie de estrategias", siendo una de ellas la "Estrategia temática para la protección del suelo" de 2006.

Previamente, y como primera etapa, el Parlamento Europeo ya había recomendado a la Comisión la elaboración de una Estrategia Temática para la Protección del Suelo en $2002^{9}$. Sin embargo, a diferencia de lo que ocurre en algún modelo de Derecho comparado, como el alemán, donde el problema de los suelos es abordado desde una perspectiva más integral, la Unión Europea viene ofreciendo hasta el momento un enfoque limitado y sectorial, centrando su perspectiva en el marco de la política ambiental (única habilitación para la adopción de medidas de utilización del suelo) y su tratamiento a efectos de su prevención y gestión de los residuos ${ }^{10}$.

En la "Estrategia temática para la protección del suelo" adoptada el 22 de septiembre de 2006 como Comunicación de la Comisión al Consejo, al Parlamento Europeo, al CESE y al Comité de las Regiones [COM (2006) 231 final], se identifica el suelo como un recurso natural, no renovable, al menos a escala humana, y se contempla la necesidad de adecuar los distintos usos a las características del suelo.

Llama la atención el interés que se muestra por preservar los suelos de alta capacidad agrícola frente a distintas presiones, incluida la urbanística, dado que los indicadores ponen de relieve que buena parte de las transformaciones del suelo se han producido a expensas de zonas agrícolas. También la solicitud a los estados miembros para la adopción de medidas que limiten su sellado. Asimismo, destaca la apuesta por la financiación de los trabajos de recuperación de suelos contaminados por antiguos usos industriales, a fin de que puedan ser urbanizados, evitando de esta manera el sellado de nuevos "terrenos vírgenes" ${ }^{11}$.

\footnotetext{
7 Sexto Programa de Acción en Materia de Medio Ambiente ("Medio Ambiente 2010: el futuro está en nuestras manos"). Decisión no. 1600/2002/CE, del Parlamento Europeo y del Consejo, de 22 de julio de 2002 (DOUE L 242, de 10.09.2002). Sus cuatro ámbitos de acción prioritarios han sido: cambio climático; biodiversidad; medio ambiente y salud; y gestión sostenible de los recursos naturales y residuos. Concluido en julio de 2012, si bien siguen aplicándose algunas de sus medidas y acciones iniciadas en el marco del mismo.

${ }^{8}$ Las Estrategias Temáticas debían ceñirse a los siguientes campos: contaminación atmosférica; prevención y reciclaje de residuos; protección y conservación del medio marino; protección del suelo; utilización sostenible de recursos naturales; y medio urbano.

${ }^{9}$ Comunicación de la Comisión [COM (2002) 179 final], de 16 de abril de 2002, hacia una Estrategia temática para la protección del suelo.

${ }^{10}$ Este enfoque habría sido confirmado por la Sentencia del Tribunal de Justicia de la Unión Europea de 7 de septiembre de 2004 (asunto C-1/03, caso Paul Van de Walle y otros y Texaco Belgium, S.A.).

${ }^{11}$ La recuperación de los suelos contaminados ha sido objeto de regulación por la Directiva 2004/35 CE del Parlamento Europeo y del Consejo, de 21 de abril de 2004, (transpuesta al Derecho español por Ley 26/2007, de 23 de octubre, de Responsabilidad Medioambiental) y la Directiva 2008/98/CE del Parlamento Europeo y del Consejo, de 19 de noviembre de 2008, por la que se derogan determinadas Directivas (transpuesta por la Ley 22/2011, de 28 de julio, de Residuos y Suelos Contaminados). Como también una de las prioridades del
} 
El documento reconoce que las disposiciones adoptadas en el contexto europeo, nacional e internacional a favor de la protección del suelo abarcan muchos ámbitos, pero que, en la medida en que su objetivo suele ser la protección de otros medios naturales o la promoción de otros objetivos, no constituyen una política coherente de protección de este recurso natural, por lo que se recomienda la adopción de una "directiva marco flexible" como el medio más adecuado para actuar.

Por ello, la Comisión elaboró también una propuesta de Directiva "por la que se establece un marco para la protección del suelo y se modifica la Directiva 2004/35/CE" [COM (2006) 232 final] en la que se incluye el establecimiento de un marco común para proteger el suelo, basándose en los principios de preservación de sus funciones, prevención y mitigación de los efectos de su degradación, restauración de los terrenos degradados e integración en otras políticas sectoriales.

El Parlamento Europeo adoptó su dictamen en primera lectura sobre la propuesta en noviembre de 2007, pero en el Consejo de Medio Ambiente de marzo de 2010 una minoría de estados miembros bloqueó la situación alegando razones de subsidiariedad, costes excesivos y cargas administrativas. Desde entonces, no se ha avanzado más en la cuestión ${ }^{12}$.

No obstante lo anterior, al margen del debate sobre la $\mathrm{PAC}^{13}$ y la Política Regional, la Comisión está desarrollando una "Asociación Europea para la Innovación sobre productividad y sostenibilidad de la agricultura", centrada especialmente en la gestión de los suelos, el uso eficiente de los recursos y la explotación sostenible de los suelos agrícolas. También trabaja en la elaboración de unas "directrices sobre mejores prácticas para limitar, mitigar y compensar el sellado de los suelos"14, a utilizar en el desarrollo del "Programa de salvaguardia de las aguas de Europa", así como en la aplicación de la Política de Cohesión ${ }^{15}$.

El debate sobre la protección del recurso suelo y sobre su racional ocupación ha tenido finalmente su reflejo en el vigente Texto Refundido de la Ley de Suelo (Real Decreto Legislativo 2/2008, de 20 de junio), que, haciéndose eco en su preámbulo de la Estrategia Territorial Europea (Postdam, 1999) y de la Comunicación de la Comisión sobre una Estrategia Temática para el Medio Ambiente Urbano [COM(2005) 718 final] -documento este último que propugna el modelo de ciudad compacta, apuesta por la regeneración de la ciudad existente frente a las nuevas transformaciones de suelo y advierte de los graves

Séptimo Programa Marco de Investigación y Desarrollo Técnico de la Unión (2007-2013). Vid. Bassols Coma (2013).

${ }^{12}$ Ello a pesar del interés mostrado por la Comisión y por otras instituciones comunitarias, como el Comité de las Regiones [vid. Dictamen sobre la aplicación de la estrategia temática para la protección del suelo (2013/C 17/08) DOUE C 17/37 19.1.2013].

${ }^{13} \mathrm{La}$ reciente reforma de la PAC, siguiendo las pautas del Reglamento (CE) no 1782/2003, establece un marco de normas de buenas condiciones agrarias y medioambientales de la tierra a partir del cual los estados miembros deben adoptar medidas nacionales atendiendo a las características específicas de las zonas respectivas, incide nuevamente en los mecanismos de prevención de la erosión del suelo, del mantenimiento de su materia orgánica y de su estructura. Vid. Reglamento (UE) no 1306/2013 del Parlamento Europeo y del Consejo, de 17 de diciembre de 2013, sobre la financiación, gestión y seguimiento de la PAC. De otro lado, estrechamente ligada con la PAC, aunque inserta en la política de medio ambiente, se encuentra también la Directiva sobre Nitratos, cuyo objetivo es reducir el contenido de nitratos de los suelos agrícolas e impedir la contaminación de los acuíferos subterráneos, problema agravado en las zonas de regadío.

14 COMISIÓN EUROPEA. Documento de trabajo de los Servicios de la Comisión. Bruselas, 15.5.2012 [SWD(2012) $101 \mathrm{final} / 2]$.

15 COMISIÓN EUROPEA. Informe de la Comisión al Parlamento Europeo, al Consejo, al Comité Económico y Social Europeo y al Comité de las Regiones "Aplicación de la Estrategia Temática para la Protección del Suelo y actividades en curso”. Bruselas, 13.2.2012 [COM(2012) 46 final]. 
inconvenientes de la urbanización dispersa y desordenada-, aborda el suelo como un "recurso económico" y como "recurso natural, escaso y no renovable", siendo uno de los mayores problemas el de la ocupación del territorio, su fragmentación y su reconversión en superficies artificiales por la expansión urbana y las infraestructuras. El preámbulo del citado Texto Refundido también recalca, entre otras cuestiones, que "todo el suelo rural tiene un valor ambiental digno de ser ponderado y la liberalización del suelo no puede fundarse en una clasificación indiscriminada, sino, supuesta una clasificación responsable del suelo urbanizable, necesario para atender las necesidades económicas y sociales" ${ }^{\text {"16. }}$

También la jurisprudencia viene dando una creciente importancia a esta cuestión, si bien de manera paulatina. Puede tomarse como ejemplo la sentencia de la Sala de lo ContenciosoAdministrativo (sección 5a) del Tribunal Supremo de 10 de julio de 2012 (Ponente: Rafael Fernández Valverde), que aplica el principio de no regresión, considerando que las decisiones que supongan una "desprotección" de suelos requiere una especial motivación adicional. Extraemos lo siguiente:

\begin{abstract}
"En este sentido, no es ocioso indicar que el carácter sostenible y medioambiental del urbanismo actual, se proyecta, de forma más directa y efectiva, en relación con los suelos rústicos en que concurren especiales valores de carácter ambiental, (...) Como ba puesto la buena doctrina española, el TRLS 08 lo que, en realidad, aporta 'es una mayor imbricación entre urbanismo y protección del medio ambiente; una especie digámoslo, de interiorización más profunda de los valores ambientales en la ordenación territorial y urbanistica, hasta hacerlos inescindibles'. (...) y que la afectación de tales suelos como consecuencia de la actuación urbanizadora proyectada se nos presenta como una actuación prácticamente irreversible, capaz. de comprometer el citado recurso natural -o su uso más racional-, además de proyectar consecuencias determinantes y nefastas para otros recursos naturales, tales como el agua, el aire o los ecosistemas. (...) Ello nos sitúa en el ámbito, propio del Derecho Medioambiental, del principio de no regresión, que, en supuestos como el de autos, implicaría la imposibilidad de no regresar de -de no poder alterar-una protección especial del terreno (...), desde luego incompatible con su urbanización, pero también directamente dirigida a la protección y conservación, frente a las propias potestades de gestión de tales suelos tanto por aplicación de su legislación especifica como por el planificador urbanistico. Este principio de no regresión, ha sido considerado como una 'cláusula de statu quo' o 'de no regresión', con la finalidad, siempre, de proteger los avances de protección alcanzados en el contenido de las normas medioambientales, con base en razones vinculadas al carácter finalista del citado derecho medioambiental, (...) En consecuencia (...) el citado principio de no regresión calificadora de los suelos especialmente protegidos implica, exige e impone un plus de motivación razonada, pormenorizada y particularizada de aquellas actuaciones administrativas que impliquen la desprotección de todo o parte de esos suelos. (...)".
\end{abstract}

\title{
4 Posibilidades de protección del suelo agrícola en la Huerta de Valencia
}

Al margen de una serie de estudios elaborados por la Generalitat sobre el recurso natural suelo y su problemática en el territorio valenciano ${ }^{17}$, entre la normativa autonómica

\footnotetext{
${ }^{16} \mathrm{El}$ artículo 2, referido al "principio de desarrollo de desarrollo territorial y urbano sostenible" propugna la protección, adecuada a su carácter, del medio rural y la preservación de los valores del suelo innecesario o inidóneo para atender las necesidades de transformación urbanística; la prevención y minimización de la contaminación de los suelos; así como la consecución de un medio urbano suficientemente dotado, "en el que se ocupe el suelo de manera eficiente".

17 Véanse las monografías editadas por la entonces Dirección General de Urbanismo y Ordenación del Territorio, de la Conselleria de Obras Públicas, Urbanismo y Transportes, de la Generalitat Valenciana:
} 
valenciana, la derogada Ley 6/1989, de 7 de julio, de la Generalitat, de Ordenación del Territorio de la Comunidad Valenciana, incluía expresamente una serie de preceptos referidos a la protección de los suelos (artículos 76-80), y concretamente a la protección de los suelos de los suelos agrícolas de alta productividad, si bien con un carácter excesivamente genérico.

Sí encontramos una mayor concreción en las Normas de Coordinación Metropolitana (NCM, adelante), dictadas en cumplimiento del mandato contenido en la Ley 5/1986, de 19 de noviembre, de la Generalitat, por la que se extinguía la Corporación Administrativa Gran Valencia ${ }^{18}$. Entre las determinaciones mínimas que el artículo 3.2 de la Ley 5/1986 preveía que podrían adoptar las NCM se incluían "las medidas de protección a adoptar en orden a la conservación del suelo, de los demás recursos naturales y a la defensa, mejora, desarrollo o renovación del medio ambiente natural y del patrimonio histórico-artístico", por lo que se incluyeron, entre otras determinaciones normas referidas a los suelos de alto valor agrícola (Norma 24) ${ }^{19}$.

En la actualidad, al margen de lo que depare en el futuro el proyecto de Ley de Ordenación del Territorio, Urbanismo y Paisaje de la Comunitat Valenciana (LOTUP) ${ }^{20}$ y de las exigencias derivadas de la evaluación ambiental de planes, programas y proyectos, y a la espera de la aprobación definitiva del PATPHV, la protección del suelo de la Huerta de Valencia debemos basarla principalmente en los criterios de ordenación territorial de la LOTPP y en las directrices contenidas en la Estrategia Territorial de la Comunitat Valenciana (ETCV, aprobada mediante Decreto 1/2011, de 13 de enero, del Consell), destacando de esta última los criterios de crecimiento y ocupación de nuevos suelos, así como la "infraestructura verde", que fue introducida de forma pionera por el legislador valenciano en la LOTPP, mediante el capítulo XIII de la Ley 12/2009, de 23 de diciembre, de la Generalitat, de Medidas Fiscales, de Gestión Administrativa y Financiera y de Organización de la Generalitat ${ }^{21}$.

Los múltiples beneficios de las infraestructuras verdes, en las que se incluyen los "terrenos que presenten especiales valores agrarios cuya preservación sea conveniente para el medio rural" (artículo 19 bis LOTPP) han sido puestos de relieve por la Comisión Europea en su Comunicación de 6 de mayo de 2013 "Infraestructura verde: mejora del capital natural de Europa"22. También por otras instancias, como el Comité de las Regiones ${ }^{23}$.

Generalitat Valenciana (1995) y Antolín Tomás (1998). También debemos referirnos a la actualización de las Cartografías Temáticas llevada a cabo por dicha Conselleria (Orden de 8 de marzo de 1999).

18 Redactadas por la entonces Conselleria de Obras Públicas, Urbanismo y Transportes y aprobadas por Decreto del Consell de 18 de julio de 1988. Estas normas nunca han sido expresamente derogadas.

${ }^{19}$ En relación a las NCM, vid. nuestro anterior trabajo: Montiel Márquez y García Tobarra (2011).

${ }^{20}$ Con posterioridad a la elaboración de esta ponencia fue finalmente aprobada la Ley 5/2014, de 25 de julio, de la Generalitat, de Ordenación del Territorio, Urbanismo y Paisaje, de la Comunitat Valenciana (LOTUP). Esta ley ha eliminado el expreso mandato contenido en el citado artículo 22.6 de la LOTPP y cualquier otra mención expresa a la Huerta de Valencia, a su protección o preservación.

${ }^{21}$ El término proviene de los EE.UU. donde el concepto es conocido como "Green Infraestructure", con la carga simbólica que conlleva la acuñación de un nuevo término. Sin embargo no es un concepto novedoso, pues en Europa ya se venía hablando de la necesidad de prever conectores funcionales o "corredores verdes", para desempeñar funciones de conexión biológica y territorial, a evitar o disminuir la fragmentación territorial, ayudando a la conexión y vertebración de espacios naturales, y cuyos ejes se constituirían por los elementos estructuradores de los sistemas hídricos y otros hitos geográficos identificables, tal como se expresa en el artículo 20, apartados 6 y 7 de la LOTPP, desde su redacción original.

22 COMISIÓN EUROPEA. Comunicación de la Comisión al Parlamento Europeo, al Consejo, al Comité Económico y Social Europeo y al Comité de las Regiones "Infraestructura verde: mejora del capital natural de Europa”. Bruselas, 6.5.2013 [COM(2013) 249 final].

23 (2013/C 356/08) DOUE C 356/43, de 5.12.2013. 
En cuanto al PATPHV, y al margen del establecimiento de "reservas de estudio", cabe recordar que no sólo renuncia a considerar alguna de las superficies de trama verde de huerta más pequeñas pero de interés que han quedado en los instersticicios de suelo actualmente sellado o edificado, sino que, además, desprotege suelo calificado actualmente como SNU protegido agrícola basándose en la información suministrada por los municipios o en diversos documentos de Concierto Previo del Plan General que carecen de informe o evaluación ambiental estratégica y que, en el mejor de los casos, obtuvieron en su día por parte de la Conselleria informe favorable condicionado expresamente a lo que resultara de los PAT de la Huerta y del Litoral (casos, por ejemplo, de Valencia y Alboraia). Todo ello sin una auténtica visión metropolitana de conjunto, y bajo la falacia de la supuesta "vocación urbana" cuando no de la antropización o degradación, sin considerar que hay situaciones fácilmente reversibles, y sin plantearse seriamente (con límites y medidas claros y concisos) las consecuencias (entendidas como presión urbanística) que tendría sobre las bolsas de suelo que se pretende proteger la urbanización de los terrenos inmediatos que el PATPHV contempla como urbanizables.

En definitiva, dado que el PATPHV no modifica ningún planeamiento municipal y respeta la ordenación prevista por los diferentes municipios (destacando el caso de Valencia), se deduce que la previsión defendida por la ETCV de "conservar como activo estratégico la mayor parte posible de suelo agricola de alta capacidad agrológica, orientando sus desarrollos urbanisticos hacia los suelos que no tengan tal consideración" (directriz 48.b) no queda garantizada posteriormente en el PATPHV. Es por ello que, con base al artículo 37.2 de la LOTPP, la ETCV podría haber incluido en su Directrices medidas concretas y efectivas para el ámbito metropolitano.

Como muestra de lo arriba expuesto, puede citarse el artículo 61.2 de la Normativa del PATPHV que indica que éste "no propone crecimiento urbanístico alguno, limitándose a orientar el desarrollo futuro mediante criterios y directrices" (...) y que "la no materialización de las previsiones de crecimiento municipal recogidas en este documento no implica su incumplimiento, sino todo lo contrario, ya que el criterio general sobre el que se sustenta el Plan de Acción Territorial es el de la mínima afección a terrenos de huerta".

Los criterios de crecimiento para suelo residencial y para actividades económicas hay que buscarlos en la ETCV, en cuya directriz 136.6 establece lo siguiente:

\section{"Los municipios del litoral clasificarán como suelo no urbanizable protegido la mayor cantidad posible de suelo de muy alta capacidad agrológica, compatiblizando dicha protección con sus necesidades de crecimiento urbanistico racionaly sostenible"}

Resulta llamativo comprobar cómo se ha eliminado de esa directriz la referencia a la incorporación de las determinaciones del PATPHV en los planes urbanísticos municipales que sí se contenía en la versión de la ETCV sometida a información pública.

Además, esta determinación tiene carácter de directriz y no de principio director, por lo que la protección de Huerta puede quedar en papel mojado, dado que el principio director es de aplicación directa y vinculante para las administraciones públicas, y las directrices son meras recomendaciones a tener en cuenta, por lo que apartarse de las mismas sólo precisa de una adecuada justificación basada en el cumplimiento de los objetivos de la ETCV y sus principios directores. 
Si a ello añadimos, por una parte, que los criterios de crecimiento de nuevo suelo urbanizable previstos en la ETCV tienen carácter orientativo, y por otro, que los índices máximos de ocupación de suelo resultante son bastante elevados (pese al factor de corrección por vertebración territorial previsto en la directriz 84), el resultado seguirá siendo el de paulatina y grave pérdida de actual suelo de Huerta en casi todos los municipios del ámbito del PATPHV, sin garantizarse en ningún momento ese crecimiento hacia el interior que la ETCV propugna ${ }^{24}$.

En resumen, después de la lectura del documento se deduce que la ETCV carece de voluntad real en orden a establecer garantías para alcanzar sus propias previsiones en el ámbito del área metropolitana de Valencia.

\section{VII Programa General de Medio Ambiente y protección del suelo}

Recientemente, mediante Decisión $\mathrm{n}^{\mathrm{o}}$ 1386/2013/UE, del Parlamento Europeo y del Consejo, de 20 de noviembre de 2013, ha sido adoptado el VII Programa General de Acción de la Unión en materia de Medio Ambiente hasta 2020, "Vivir bien, respetando los limites de nuestro planeta" (DOUE L 354, de 28.12.2013.), texto que pone especial énfasis en la problemática de la degradación del suelo. El documento recuerda también que los estados miembros han obtenido desiguales resultados en esta materia.

El nuevo Programa, ahora General al tratar de superar el estricto sector ambiental e integrar plenamente las consideraciones medioambientales en otras políticas, pretende basarse en las iniciativas adoptadas en el marco de la Estrategia Europa 2020, y relaciona nueve "objetivos prioritarios", para los que, tras describir la situación y los problemas de partida, y las medidas adoptadas, apunta una serie de medidas necesarias a abordar.

Respecto a nuestro objeto de estudio, nos interesa en este punto principalmente lo previsto en los objetivos $n^{\circ} 1$ a 3,7 y 8 . Al objetivo $n^{\circ} 6$ nos referiremos en el siguiente epígrafe ${ }^{25}$.

El primer objetivo prioritario ("proteger, conservar y mejorar el capital natural de la UE") incluye una expresa mención a las presiones ejercidas sobre las aguas dulces, a la protección del recurso suelo (especialmente los suelos fértiles agrícolas) y al problema de la gestión de nitrógeno y fósforo como nutrientes.

Ante la creciente urbanización y sellado de suelo, considera necesaria la adopción por los estados miembros de decisiones más sostenibles en materia de ordenación del territorio. Por otra parte, en resumen, se establecerán metas en relación con el suelo y el uso sostenible de la tierra, y se ampliará la utilización de la infraestructura verde para paliar la fragmentación de los terrenos, medida esta última también prevista en el objetivo $\mathrm{n}^{\circ} 3$ ("proteger a los ciudadanos de la UE de las presiones y riesgos medioambientales para la salud y el bienestar').

\footnotetext{
24 Sobre el PATPHV y la ETE, vid. Burriel de Orueta (2009).

${ }^{25} \mathrm{El}$ resto de objetivos son los siguientes: $\mathrm{n}^{\circ}$ 4: maximizar los beneficios de la legislación de medio ambiente de la UE; $n^{\circ}$ 5: mejorar la base de información de la política de medio ambiente; y no 9: reforzar la eficacia de la UE a la hora de afrontar los desafíos ambientales a nivel regional y mundial. Vid. Galera Rodrigo y Hernández-Bataller (2013).
} 
El segundo objetivo persigue "convertir a la UE en una economía bipocarbónica, eficiente en el uso de los recursos, ecológica y competitiva". Entre otras determinaciones, se pretende mejorar la eficiencia hídrica y reducir para 2050 las emisiones de GEI en un 80-95\% en comparación con 1990.

$\mathrm{La}$ infraestructura verde vuelve a citarse en el objetivo prioritario $\mathrm{n}^{\circ} 7$ ("intensificar la integración medioambiental y la coherencia entre políticas"), señalándose que la incorporación de la misma en planes y programas conexos puede contribuir a superar la fragmentación de hábitats y a preservar o restaurar la conectividad ecológica. Además de esta medida, se prevé que las políticas sectoriales de la UE y los estados miembros se desarrollen y apliquen de tal forma que favorezcan la consecución de objetivos y metas pertinentes en materia de clima y medio ambiente.

Por último, en cuanto al objetivo prioritario $\mathrm{n}^{\circ} 8$ ("aumentar la sostenibilidad de las ciudades de la UE'), el Programa garantizará que, para 2020 (apartado 91): la mayoría de las ciudades de la UE estén aplicando políticas de ordenación y diseño sostenibles del espacio urbano, siendo necesario, en particular: determinar y acordar un conjunto de criterios para evaluar el comportamiento ambiental de las ciudades.

\section{La nueva regulación de los instrumentos financieros de la UE y su posible incidencia en la Huerta de Valencia}

No debemos olvidar el importante desarrollo que han experimentado durante los últimos años las medidas de la Unión para la financiación de actuaciones medioambientales ${ }^{26}$, entre las que cabe destacar el Instrumento Financiero para el Medio Ambiente ("L'Instrument Financier pour l'Environnement" o LIFE), establecido en 1992, y que, a diferencia de los fondos estructurales o del Fondo de Cohesión (que también atiende acciones medioambientales), sí opera en toda la Unión Europea.

La financiación de actuaciones medioambientales adquiere mayor importancia si cabe en la medida en que la nueva programación de los fondos estructurales (2014-2020) está concebida como el instrumento financiero para la consecución de los objetivos de la Estrategia Europa 2020, siendo una de sus líneas estratégicas el "crecimiento sostenible".

Precisamente el objetivo prioritario n ${ }^{\circ} 6$ del VII Programa General de Acción en materia de Medio Ambiente pretende "asegurar inversiones para la politica en materia de clima y medio ambiente y fijar correctamente los precios", suprimiendo progresivamente las subvenciones perjudiciales para el medio ambiente, intensificando la aplicación de instrumentos de mercado, en particular impuestos, tarifas y tasas, y ampliando los mercados de bienes y servicios ambientales, teniendo debidamente en cuenta cualquier impacto social negativo.

En cuanto a la agricultura, recuerda que la Comisión ha propuesto ofrecer mayores incentivos a los agricultores para que suministren bienes y servicios públicos beneficiosos para el medio ambiente (si bien las "directrices aplicables a las ayudas estatales en los sectores agrícola

\footnotetext{
${ }^{26}$ Existen ayudas económicas que, pese a no encuadrarse en la protección del medio ambiente, sí presentan aspectos medioambientales, como la financiación de algunos programas de investigación y de enseñanza; los préstamos y las facilidades de financiación otorgados por el Banco Europeo de Inversiones (BEI); las ayudas financieras a determinadas acciones con cargo al FEDER y al Fondo de Cohesión; y los fondos de la PAC orientados al desarrollo rural sostenible (FEADER).
} 
y forestal y en las zonas rurales de 2014 a 2020" publicadas recientemente por la Comisión no se hacen eco de la específica problemática periurbana ${ }^{27}$ ).

A su vez, el VII Programa señala también que la inclusión de "proyectos integrados" en el programa LIFE permitirá la combinación de fondos con prioridades políticas de una manera más estratégica y eficiente.

Tras el TFUE, la competencia rebautizada como política de "Cohesión Económica, Social y Territorial" habilita a la Unión Europea a desarrollar acciones encaminadas a reforzar la cohesión en su triple vertiente (económica, social y territorial), con el fin de paliar los desequilibrios existentes dentro del territorio de la Unión y de promover un desarrollo armonioso del conjunto del mismo.

Actualmente, esta competencia comunitaria, que se enmarca dentro de la concepción amplia de la ordenación del territorio, tiene una gran repercusión en algunos ámbitos territoriales de la Unión, teniendo en ocasiones mayor peso económico que muchas de las acciones de política regional llevadas a cabo por los estados miembros o por las propias instancias administrativas regionales.

En la actualidad nos encontramos ante un nuevo período de programación 2014-2020, en el que se han introducido nuevas prioridades de inversión de política regional, en coherencia con la Estrategia Europea 2020. Una de las novedades es la más estrecha coordinación entre los distintos fondos, para evitar solapamientos y mejorar su eficacia.

De este modo, el reciente Reglamento (UE) no 1303/2013, del Parlamento Europeo y del Consejo, de 17 de diciembre de $2013^{28}$, establece normas comunes relativas al FEDER ${ }^{29}$, al FSE, al Fondo de Cohesión, al FEADER y al FEMP, y ha obligado a modificar varios Reglamentos.

A través de algunos de estos fondos, la Unión Europea impone sus propias directrices (no vinculantes) al ejercicio de las competencias de los estados miembros.

La Política Regional es concebida ahora como mecanismo para lograr una economía competitiva que use eficazmente los recursos, con pocas emisiones de carbono y resistente al cambio climático. Los fondos con finalidad estructural han de servir de motor de crecimiento y competitividad, resultando ser factores claves de este crecimiento las PYMES, la eficiencia energética y la innovación. En resumen, la nueva estrategia apoya en dos pilares: el primero, que se resume en la máxima "más inversión en crecimiento sostenible", y el segundo ("mejor inversión"), centrado especialmente en la contratación pública ${ }^{30}$.

Tras la ampliación a los países de la Europa oriental y central, en el contexto de una Unión Europea de 28 estados miembros, por el llamado "efecto estadístico", el Reino de España ha visto modificada la clasificación de sus territorios a efectos de política regional europea,

27 COMISIÓN EUROPEA. Directrices de la Unión Europea aplicables a las ayudas estatales en los sectores agrícola y forestal y en las zonas rurales de 2014 a 2020 (2014/C 204/01). DOUE C 204/1, de 1.7.2014.

${ }^{28}$ DOUE L 347, de 20.12.2013.

${ }^{29}$ Como novedad, destaca el principio de concentración de las actuaciones del FEDER. Atendiendo a la categoría de las regiones beneficiarias, las ayudas se concentrarán en investigación e innovación, PYMES y reducción del cambio climático.

30 Galera Rodrigo (2014). 
si bien se han previsto instrumentos transitorios para que las regiones que, como la valenciana, venían recibiendo ayudas no las pierdan de forma abrupta. Ahora se distinguen tres categorías de regiones: menos desarrolladas (PIB per cápita inferior al 75\% de la media UE), en transición (PIB per cápita entre el 75 y el $90 \%$ de la media UE) y más desarrolladas (PIB per cápita superior al 90\% de la media UE). La Comunitat Valenciana se encuentra en el tercer grupo.

Cabe destacar que una de las novedades del nuevo período de programación es la concepción de las ciudades como motores de la economía de Europa. Para ello se refuerza su papel en el contexto de la Política Regional. También que el objetivo prioritario $\mathrm{n}^{\circ} 8$ del VII Programa ("aumentar la sostenibilidad de las ciudades de la UE"), ha considerado necesario garantizar que las ciudades dispongan de información sobre la financiación de medidas para mejorar la sostenibilidad urbana, y que tengan acceso a esos fondos.

Consecuentemente, se ha procedido a asignar un porcentaje mínimo de fondos para financiar acciones de desarrollo urbano sostenible y se crea la nueva herramienta de "Inversión Territorial Integrada" (ITI).

En resumen, consideramos que debería aprovecharse la adopción del Marco Estratégico Común (MEC) y el Acuerdo de Asociación para los Fondos Estructurales y de Inversión Europeos 2014-2020 para tratar de apoyar nuevas acciones a favor de l'Horta de Valencia como elemento protagonista y fundamental de la infraestructura verde del área metropolitana de Valencia.

\section{Algunas conclusiones}

- Constatamos la inexistencia de una política europea (ni nacional) específica en materia de agricultura periurbana. Esta realidad presente en numerosas grandes ciudades europeas no ha merecido, hasta el momento, una atención adecuada ni en las acciones orientadas al desarrollo urbano sostenible ni en las referentes a desarrollo rural.

- Las acciones comunitarias específicas en materia del suelo como recurso natural, han atendido fundamentalmente a la problemática de los suelos contaminados, encontrándose a faltar una visión más integrada, acorde a la multiplicidad de sus funciones.

- La noción de desarrollo rural, tal y como se concibe en la PAC y en los fondos con finalidad estructural, resulta en exceso limitante, ignorando situaciones nuevas, consecuencia del desarrollo urbanístico, industrial, terciario y de infraestructuras de comunicación y transporte, que fagocita el espacio productivo agrario próximo, generando disfunciones y conflictos entre los usos presentes, además de dar lugar a nuevos paisajes rururbanos los cuales precisan de una particular atención y especial tratamiento.

- No puede desconocerse además que la agricultura periurbana presenta valores añadidos en orden a la economía local, el medio ambiente, la salud y el paisaje.

- Resulta evidente que la agricultura periurbana puede jugar un importante papel en orden a las infraestructuras verdes adecuado al VII Programa General de Medio Ambiente.

- En definitiva, la agricultura periurbana constituye una realidad europea valiosa y sometida a peculiares tensiones y dinámicas que reclama un lugar propio en las 
políticas de cohesión territorial y de desarrollo agrario, tal y como ahora disfrutan otras realidades como, por ejemplo, ocurre con la agricultura de montaña.

- En lo que al caso valenciano respecta, resulta especialmente lacerante la renuncia de las autoridades valencianas no sólo a cumplir sus propios compromisos, expresados en diversas normas y planes, si no también su incapacidad para exigir del gobierno central una consideración y tratamiento adecuado para la agricultura periurbana y la reivindicación de decisiones y fondos estructurales para tal fin a la Unión Europea.

\section{Referencias}

Antolín Tomás, C. (coord.). (1998): El suelo como recurso natural en la Comunidad Valenciana Generalitat Valenciana, Conselleria de Obras Públicas, Urbanismo y Transportes (COPUT). Valencia.

Bassols Coma, M. (2013): El Derecho urbanístico español y el Derecho de la Unión Europea. Revista de Derecho Urbanístico y Medio Ambiente (RDUyMA), núm. 285, 2013.

Burriel de Orueta, E. L. (2009): La planificación territorial en la Comunidad Valenciana (1986- 2009). Scripta Nova. Revista Electrónica de Geografía y Ciencias Sociales. Universidad de Barcelona. Vol. XIII, núm. 306, 1 de diciembre de 2009.

Camacho Ballesta, J.A. y Melikhova, Y. (2010): Perspectiva territorial de la Unión Europea: el largo camino hacia la cohesión territorial. Cuadernos Geográficos, 47 (2010-2).

Farinós Dasí, J. (2009): 'Bases, métodos e instrumentos para el desarrollo y la cohesión territoriales. Diagnóstico y propuestas para el debate y la acción, Farinós, J., Romero, J., y Salom, J. (eds.) Cohesión e inteligencia territorial. Dinámicas y procesos para una mejor planificación y toma de decisiones. Publicacions de la Universitat de València. Valencia.

Galera Rodrigo, S. (2014): Del ahorro de energía a la eficiencia energética: objetivos e instrumentos de las políticas europeas. RDUyMA, núm. 289, 2014.

Galera Rodrigo, S. y Hernández-Bataller, B. (2013): 'Crónica Europea VI. RDUyMA, núm. 286, 2013.

Generalitat Valenciana, COPUT. (1995): El uso del suelo en la Comunidad Valenciana. Documento III del Avance del PDU (Plan de desarrollo urbanistico de la Comunidad Valenciana). Valencia.

Giobellina, B. L. (2012): Procesos emergentes: de la Huerta Andalusí a la Huerta agroecológica del siglo XXI, Romero González, J. y Francés, M. (eds.). La Huerta de Valencia. Un paisaje cultural con futuro incierto. Publicacions de la Universitat de València. Valencia.

Montiel Márquez, A. (2012): Expansión metropolitana y agricultura periurbana. Tensiones y oportunidades en L'Horta de València, Romero González, J. y Francés, M. (eds.). “La Huerta de Valencia. Un paisaje cultural con futuro incierto". op. cit. 
Montiel Márquez, A. y García Tobarra, E. (2011): L’Horta de València. Una realidad metropolitana ignorada, Actes del III Congrés d'Estudis de l'Horta Nord, vol. II. Universitat Politècnica de València. València.

Tortosa, F. (2007): La comarca de l'Horta: El cap i casal devora l'Horta fértil que l'envolta, en Mètode, Revista de difusió de la investigació, núm. 53. Universitat de València. Valencia. 